\title{
The significance of anti-DFS70 antibodies in the diagnosis of autoimmune disorders
}

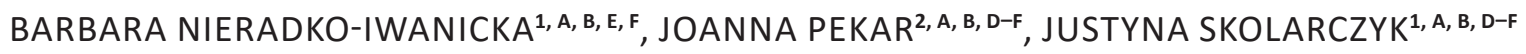 \\ ${ }^{1}$ Chair and Department of Hygiene, Medical University of Lublin, Poland \\ ${ }^{2}$ Students' Scientific Association at the Chair and Department of Hygiene, Medical University of Lublin, Poland
}

A - Study Design, B - Data Collection, C - Statistical Analysis, D - Data Interpretation, E - Manuscript Preparation, F - Literature Search, G - Funds Collection

\begin{abstract}
Summary Background. Anti-DFS70 antibodies are a subgroup of antinuclear antibodies (ANA). They are connected with the dense fine speckled autoantigen of $70 \mathrm{kD}$, known as the lens epithelium-derived growth factor $\mathrm{p} 75$.

Objectives. The objective of the review is to present the role of anti-DFS70 antibodies in the diagnosis of autoimmune diseases on the basis of recent publications.

Material and methods. The authors searched for articles in the Pubmed database using the key words: anti-DFS70 antibodies, systemic autoimmune rheumatic diseases and autoimmune disorders.

Results. Anti-DFS70 antibodies can be detected in eye diseases, atopic diseases, alopecia areata, fibromyalgia, asthma, chronic fatigue syndrome, tumors, Hashimoto's disease, Graves' disease, Behcet's disease, inflammatory bowel diseases, neoplasms and in infectious diseases. These antibodies are sometimes detected in patients with ANA-associated rheumatic diseases (AARD). Up to $20 \%$ of serum samples from healthy individuals (HI) are ANA-positive. This is probably due to the presence of monospecific anti-DFS70 antibodies. Monospecific anti-DFS70 antibodies are not associated with AARD, but mixed anti-DFS70 can be found in AARD.

Conclusions. Family physicians usually do not order ANA, extractable nuclear antigens (ENA) or anti-DFS70 antibodies, but they should be aware that anti-DFS70 antibodies are biomarkers that can discriminate AARD from non-AARD, save patients from unnecessary, potentially toxic treatment and save finances typically spent on retesting and visits to specialists.

Key words: anti-DFS-70 antibodies, autoimmune disorders, systemic autoimmune rheumatic diseases.
\end{abstract}

Nieradko-Iwanicka B, Pekar J, Skolarczyk J. The significance of anti-DFS70 antibodies in the diagnosis of autoimmune disorders. Fam Med Prim Care Rev 2017; 19(2): 173-176, doi: https://doi.org/10.5114/fmpcr.2017.67874.

\section{Background}

Antinuclear antibodies (ANAs) are a hallmark of ANA-associated rheumatic disease (AARD), such as: systemic lupus erythematosus (SLE), mixed connective tissue disease (MCTD), Sjögren's syndrome (SjS), polymyositis/dermatomyositis (PM/ /DM) and systemic sclerosis (SSc) [1, 2]. ANAs target protein, and nucleic acid antigens are predominantly localized in the cell nucleus. Detection of ANA is the first phase of laboratory diagnostics of AARD. Understanding the mechanisms underlying the generation of ANAs in AARD and other chronic inflammatory conditions remains a challenge.

A relatively common serum ANA pattern was observed in numerous clinical and research laboratories and was characterized by staining of dense fine speckles in the nucleus, with strong staining of mitotic chromosomes. Human sera displaying this pattern by indirect immunofluorescence (IIFA) react by immunoblotting with a band of $\sim 70 \mathrm{kD}$. The nuclear autoantigen was designated as the dense fine speckled 70 antigen (DFS-70). The IIFA on Hep- 2 cells and chemiluminescence anti-DFS70 assay $(\mathrm{CIA})$ are the most commonly used methods of their detection. However, a novel immunoadsorption method for identification of anti-DFS70 was described by Bentow et al. [3].

The anti-DFS70 antibodies were identified in 2000. They belong to ANA. They can connect with the dense fine speckled autoantigen of $70 \mathrm{kD}$ (DFS-70), known as the lens epithelium- -derived growth factor p75 (LEDGFp75) [4]. DFS-70 is highly expressed in prostate tumor tissues [5]. DFS-70 serves as a cofactor to human immunodeficiency virus (HIV) replication, interfering with its integrase [6].

There is data that anti-DFS70 can discriminate AARD from non-AARD [7]; therefore, it is important for family physicians to be aware of the significance of anti-DFS70.

\section{Objectives}

The objective of the review is to present the role of anti-DFS70 in the diagnosis of autoimmune diseases on the basis of recent publications.

\section{Material and methods}

The authors searched for articles in the Pubmed database using the key words: anti-DFS70 antibodies and autoimmune disorders, as there were more records identified than in the Web of Science. For the query 'search anti-DFS70 antibodies and autoimmune disorders', 16 items were found; for 'search autoimmune disorders' - 449,431; for 'search anti-DFS70 antibodies' - 101, for 'search anti-DFS70 antibodies, autoimmune disorders' -1 item. The number of records after duplicates were removed was 449,330 . The number of full-text articles in this field eligible to be cited in this review was 30 (Figure 1). 


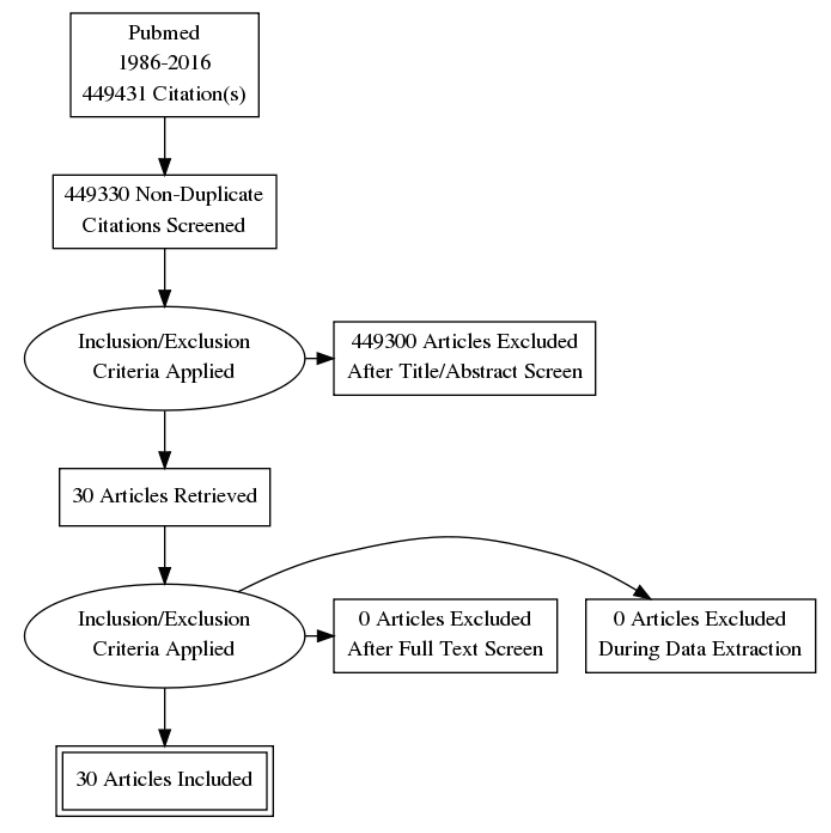

Figure 1. Prisma flow diagram

\section{Autoimmune disorders}

Autoimmune disorders are diagnoses in the presence of autoantibodies or evidence of cellular reactivity to a self, autoantibody or lymphocytic infiltrate, as well as if it is possible to demonstrate that relevant autoantibody or T cells can cause pathology. An indirect presumption of an autoimmune disorder can be made if there is a beneficial effect from immunosuppressive agents, if there is no evidence of infection or other cause of disease and if there is an association with further evidence of autoimmunity. Supportive evidence of an autoimmune disease may be deduced from reasonable animal models. There is a wide spectrum of autoimmune diseases, and some specifically affect a single organ (Grave's disease, vitiligo, Hashimoto's thyroiditis, autoimmune polyglandular syndrome, autoimmune hemolytic anemia, autoimmune thrombocytopenic purpura, type 1 diabetes mellitus, pernicious anemia, myasthenia gravis, multiple sclerosis, Guillian-Barré syndrome, pemphigus vulgaris, stiff-person syndrome, autoimmune alopecia, Goodpasture's syndrome). Many autoimmune diseases are systemic disorders with the involvement of many organs (SLE, MCTD, SjS, PM/DM, $\mathrm{SSc}$, rheumatoid arthritis, systemic necrotizing vasculitis, granulomatosis with polyangiitis, antiphospholopid syndrome) [1, 2].

\section{Characteristics of anti-DFS70 autoantibodies}

Several publications show that anti-DFS70 antibodies can be present in AARD [2-4, 7].

DFS-70/LEDGFp75 can be detected in different human tissues, in fibroblasts, keratinocytes and cancer cells [8]. DFS-70/ /LEDGFp75 is encoded by the PS1P1/LEDGF gene, which consists of 15 exons and 14 introns [9]. The C-terminus of DFS-70/LEDGFp75 contains the autoepitope (aa 347-429), recognized by the autoantibodies [10]. DFS-70/LEDGFp75 can act as a stress survival protein [11]. It can protect from environmental factors that induce cellular stress, such as certain chemotherapeutic drugs, hyperthermia, alcohol, nutrient deprivation, ultraviolet B (UVB) irradiation and hydrogen peroxide $[9,12,13]$. Oxidative stress caused by these factors induces upregulation and activation of DFS-70/LEDGFp75. Some studies suggested that when DFS-70/ /LEDGFp75 is upregulated and activated, it regulates stress gene expression [14]. Anti-DFS70 antibodies may lead to depletion of this protein. Transient depletion of this protein can cause decreased cell survival, whereas selection of stable cell clones that survived the transient depletion of this protein yields viable cells that have developed compensatory mechanisms to survive with limited DFS-70/LEDGF/p75 [15, 16]. Anti-DFS70 antibodies are manly IgG antibodies.

\section{Prevalence of anti-DFS70 antibodies in different diseases}

Most often, anti-DFS70 antibodies are detected in Vogt-Koyanagi-Harada syndrome (characterized by acute bilateral panuveitis, vitiligo, alopecia, meningitis and loss of hearing) (66.7\%) [17]. They are detected in patients with different autoimmune diseases: alopecia areata (19.8\%), asthma (16\%), atopic dermatitis (IgE and IgG4) (0-71\%), Behçet's disease (34.4\%), Grave's disease (1.7\%), Hashimoto's thyroiditis (6\%), uveitis (14.3\%), localized scleroderma (13.8\%), psoriasis $(4.5 \%)$, sarcoidosis (25\%) [18-21]. Anti-DFS70 antibodies can also be detected in patients with neoplasms: prostate cancer (17.2-22.3\%) [18-20, 22], acute myelogenous leukemia [22], chronic myeloid leukemia [23], mixed-lineage leukemia [24], colon, thyroid, breast cancer, liver and uterine tumors [22]. Anti-DFS70 antibodies are detected in patients with AARD (on average in 2-3\%) [3]: SLE (0-5.7\%), SSc (0-5.7\%), SjS (0-28.6\%), rheumatoid arthritis (RA) (0-2.6\%), DM (0-6.4\%) [20, 25, 26]. Increased titers of anti-DFS70 antibodies were detected in children with chronic fatigue syndrome, but not in children with chronic fatigue syndrome and fibromyalgia. In adults with chronic fatigue syndrome, these antibodies were rarely detected [4]. Anti-DFS70 antibodies are more likely to develop in children and young individuals [21].

\section{Prevalence of anti-DFS70 antibodies in healthy individuals}

Watanabe et al. reported on the frequency of anti-DFS70 autoantibodies in a study involving 597 healthy hospital workers in Japan and 200 patients with AARD. The main conclusion of their report was that anti-DFS70 autoantibodies are common in healthy individuals, but not in patients with rheumatic diseases. The frequency of anti-DFS70 antibodies was higher in women, and their frequency decreases with increasing age. Watanabe et al. suggested that anti-DFS70 antibodies could be defined as natural autoantibodies, which are antibodies present in the sera of healthy individuals in the absence of deliberate immunization with any antigen. They also concluded that the presence of anti-DFS70 autoantibodies could rule out systemic autoimmune disease, saving costs on diagnostic tests [21].

On the other hand, the study of Ochs et al. indicated that anti-DFS70 antibodies occur in a variety of chronic inflammatory conditions (atopic dermatitis, asthma, interstitial cystitis, SjS, psoriasis, SSc, chronic fatigue syndrome) [4]. Anti-DFS70 antibodies can also be detected in thrombotic disease and obstetric complications [27]. Several studies have shown that up to $20 \%$ of serum samples from healthy individuals $(\mathrm{HI})$ are ANA-positive. This is probably due to the presence of monospecific anti-DFS70 antibodies [21, 28-30]. To a certain degree, the discrepancies in the frequencies of anti-DFS70 antibodies result from inter-laboratory differences in detection platforms, human expertise in the detection of these antibodies and use of multiple methods for their detection $[3,17,20,28]$.

\section{Predictive value of anti-DFS70 antibodies}

In light of these somewhat conflicting studies, Gundin et al. asked what the anti-DFS70 antibodies tell us [7]. In their study, none of the patients with positive monospecific anti-DFS-70 developed AARD during a 10-year follow up. The patients $(n=181)$ were positive for ANA, but with no extractable nuclear antigen (ENA) reactivity. Their sera were tested for anti-DFS70 antibodies. The study compared the cost effectiveness of the conventional algorithm used in the diagnostics of ANA-positive patients with a new algorithm including anti-DFS70 antibody detection 
in ANA-positive and ENA-negative patients. In the conventional algorithm, every ANA-positive patient had ENA and anti-dsDNA done. If they were negative, a periodic follow up and repetition of ANA and ENA were recommended, which generated financial costs. In the new algorithm including anti-DFS70 antibody detection in ANA-positive and ENA-negative patients, a test for anti-DFS70 was performed. If the patient's serum was anti-DFS70 positive, non-AARD was diagnosed and no repetitions of ANA or ENA were recommended. Using the anti-DFS70 criteria, it is possible to achieve a $70 \%$ decrease in outpatient clinic visits, $75 \%$ for rheumatologists and $30 \%$ for other specialists [7]. It is worth considering including anti-DFS70 as exclusion criteria in SLE, especially as Systemic Lupus Collaborating Clinics (SLICC) and the American College of Rheumatology (ACR) SLE criteria contain 'ANA-positive': an abnormal titer of ANA by immunofluorescence or an equivalent assay at any point in time in the absence of drugs known to induce ANA (procainamide, propafenone, disopyramide, hydralazine, angiotensin-converting enzyme inhibitors, beta blockers, propylthiouracil, chlorpromazine, lithium, carbamazepine, phenytoin, isoniazid, minocycline, sulfasalazine hydrochlorothiazide, lovastatin, simvastatin, interferons, ANA inhibitors). ANAs are considered to be the best screening test for SLE with $98 \%$ prevalence. Repeated negative tests make SLE unlikely. ANA-negative SLE exists, but it is rare in adults and is usually associated with other autoantibodies (anti-dsDNA or anti-Ro). Anti-dsDNA is present in $70 \%$ of SLE patients, anti-Sm in $25 \%$, anti-RNP in $40 \%$, anti-Ro in $30 \%$, anti-La in $10 \%$, antiphospholipid in 50\%, antineural in $60 \%$ and antiribosomal $\mathrm{P}$ in $20 \%$.

\section{Indications for testing for anti-DFS70 antibodies}

ANA-positive patients should be tested for anti-DFS70 antibodies. In this manner, the risk of inappropriate treatment for AARD might be reduced, and the health care system avoids unnecessary repeat testing for ANA, as well as referring patients to specialists.
Family physicians do not routinely screen their patients for antinuclear antibodies. This is the expertise of specialists. However, taking into consideration the long lines to rheumatologists, immunologists and other specialists who often order and interpret ANA and/or ENA together with the devastating effects of untreated SLE, MCTD, SjS, PM/DM and SSc, it is worth remembering that having these laboratory tests done with highly positive results family physicians have the right to refer their patients with a 'cito!' mark. However, the initial visit to a specialist is usually an interview, looking for events in their medical history that could fulfill some criteria of AARD, such as pleural effusion, nephritis, hypersensitivity to ultraviolet light in SLE, eye and mouth dryness in SjS, miscarriage in antiphospholipid syndrome or morning stiffness in RA. This is followed by a physical examination, looking for swollen joints in RA, malar rash or other dermatologic manifestations of SLE, the ' $V$ ' sign and weak muscles in PM/DM, skin hardening, microstomia, teleangiectasiae, Raynaud's sign and wrinkles around the mouth in SSc, enlarged salivary glands in SjS and livedo reticularis in antiphospholipid syndrome. Eventually, the rheumatologists orders ANA and/or ENA, urinalysis, blood morphology and biochemistry. Waiting for these analyses until the next visit may be too long for many, and delayed treatment may not be fully effective. On the other hand, overtreatment of those who were underdiagnosed may lead to harmful side effects. Therefore, it makes sense, in the case of clinical suspicion of AARD, to order an anti-DFS70 antibody in ANA-positive and ENA-negative patients.

\section{Conclusions}

Family physicians do not usually order ANA, ENA nor anti-DFS70 antibodies, but they should be aware that anti-DFS70 antibodies are biomarkers that can discriminate AARD from non-AARD, save patients from unnecessary, potentially toxic treatment and save finances typically spent on retesting and visits to specialists.

Source of funding: The development of this paper was financed by the Medical University of Lublin in 2016.

Conflict of interest: The authors declare no conflict of interests.

\section{References}

1. Tan EM. Autoantibodies and autoimmunity: a three-decade perspective. A tribute to Henry G. Kunkel. Ann NY Acad Sci 1997; 815: $1-14$.

2. Seelig CA, Bauer O, Seelig HP. Autoantibodies against DFS70/LEDGF exclusion markers for Systemic Autoimmune Rheumatic Diseases (SARD). Clin Lab 2016; 62(4): 499-517.

3. Bentow C, Rosenblum R, Correia P, et al. Development and multi-center evaluation of a novel immunoadsorption method for antiDFS70 antibodies. Lupus 2016; 25(8): 897-904.

4. Ochs RL, Muro Y, Si Y, et al. Autoantibodies to DFS $70 \mathrm{kd} /$ transcription coactivator p75 in atopic dermatitis and other conditions. J Allergy Clin Immunol 2000; 105(6 Pt 1): 1211-1220.

5. Daniels T, Zhang J, Gutierrez I, et al. Antinuclear autoantibodies in prostate cancer: immunity to LEDGF/p75, a survival protein highly expressed in prostate tumors and cleaved during apoptosis. Prostate 2005; 62(1): 14-26.

6. Maertens G, Cherepanov P, Pluymers W, et al. LEDGF/p75 is essential for nuclear and chromosomal argeting of HIV-1 integrase in human cells. J Biol Chem 2003; 278(35): 33528-33539.

7. Gundín S, Irure-Ventura J, Asensio E, et al. Measurement of anti-DFS70 antibodies in patients with ANA-associated autoimmune rheumatic diseases suspicion is cost-effective. Auto Immun Highlights 2016; 7(1): 10, doi: 10.1007/s13317-016-0082-1.

8. Singh DP, Ohguro N, Chylack LT Jr, et al. Lens epithelium-derived growth factor: increased resistance to thermal and oxidative stresses. Invest Ophthalmol Vis Sci 1999; 40(7): 1444-1445.

9. Singh DP, Kimura A, Chylack LT Jr, et al. Lens epithelium-derived growth factor (LEDGF/p75) and p52 are derived from a single gene by alternative splicing. Gene 2000; 242(1-2): 265-273.

10. Ogawa Y, Sugiura K, Watanabe A, et al. Autoantigenicity of DFS70 is restricted to the conformational epitope of C-terminal alpha-helical domain. J Autoimmun 2004; 23(3): 221-231.

11. Brown-Bryan TA, Leoh LS, Ganapathy V, et al. Alternative splicing and caspase-mediated cleavage generate antagonisticvariants of the stress oncoprotein LEDGF/p75. Mol Cancer Res 2008; 6(8): 1293-1307.

12. Shinohara T, Singh DP, Fatma N. LEDGF, a survival factor, activates stress-related genes. Prog Retin Eye Res 2002; 21(3): $341-358$.

13. Sharma P, Singh DP, Fatma LT, et al. Activation of LEDGF gene by thermal- and oxidative-stresses. Biochem Biophys Res Commun 2000; 276(3): 1320-1324.

14. Basu A, Drame A, Muñoz R, et al. Pathway specific geneexpression profiling reveals oxidative stress genes potentially regulated by transcription co-activator LEDGF/p75 in PCa cells. Prostate 2012; 72(6): 597-611.

15. Llano M, Saenz DT, Meehan A, et al. An essential role for LEDGF/p75 in HIV integration. Science 2006; 314(5798): 461-464. 
16. Vandekerckhove L, Christ F, Van Maele B, et al. Transient and stable knockdown of the integrase cofactor LEDGF/p75 reveals its role in the replication cycle of human immunodeficiency virus. J Virol 2006; 80(4): 1886-1896.

17. Ochs RL, Mahler M, Basu A, et al. The significance of autoantibodies to DFS70/LEDGFp75 in health and disease: integrating basic science with clinical understanding. Clin Exp Med 2016; 16(3): 273-293.

18. Daniels T, Zhang J, Gutierrez I, et al. Antinuclear autoantibodies in PCa: immunity to LEDGF/p75, a survival protein highly expressed in prostate tumors and cleaved during apoptosis. Prostate 2005; 62(1): 14-26.

19. O'Rourke DJ, DiJohnson DA, Caiazzo RJ Jr, et al. Autoantibody signatures as biomarkers to distinguish PCa from benign prostatic hyperplasia in patients with increased serum prostate specific antigen. Clin Chim Acta 2012; 413(5-6): 561-567.

20. Conrad K, Röber N, Andrade LE, et al. The clinical relevance of anti-DFS70 autoantibodies. Clin Rev Allergy Immunol 2016; 52(2): 202-216, doi: 10.1007/s12016-016-8564-5.

21. Watanabe A, Kodera M, Sugiura K, et al. Anti-DFS70 antibodies in 597 healthy hospital workers. Arthritis Rheum 2004; 50: 892-900.

22. Huang TS, Myklebust LM, Kjarland E, et al. LEDGF/p75 has increased expression in blasts from chemotherapy-resistant human acute myelogenic leukemia patients and protects leukemia cells from apoptosis in vitro. Mol Cancer 2007; 6: 31.

23. Grand FH, Koduru P, Cross NC, et al. NUP98-LEDGF fusion and $\mathrm{t}(9 ; 11)$ in transformed chronic myeloid leukemia. Leuk Res 2005; 29(12): 1469-1472.

24. Méreau H, De Rijck J, Čermáková K, et al. Impairing MLL-fusion gene-mediated transformation by dissecting criticalinteractions with the lens epithelium-derived growth factor (LEDGF/p75). Leukemia 2013; 27(6): 1245-1253.

25. Mahler M, Parker T, Peebles CL, et al. Anti-DFS70/LEDGF antibodies are more prevalent in healthy individuals compared to patients with systemic autoimmune rheumatic diseases. J Rheumatol 2012; 39(11): 2104-2110.

26. Muro Y, Sugiura K, Nakashima R, et al. Low prevalence of anti-DFS70/LEDGF antibodies in patients with dermatomyositis and other systemic autoimmune rheumatic diseases. J Rheumatol 2013; 40(1): 92-93.

27. Marlet J, Ankri A, Charuel JL, et al. Thrombophilia associated with antiDFS70 autoantibodies. PLoS One 2015; 10(9): e0138671, doi: 10.1371/journal.pone.0138671.

28. Mahler M, Meroni PL, Andrade LE, et al. Towards a better understanding of the clinical association of anti-DFS70 autoantibodies. Autoimmun Rev 2016; 15(2): 198-201, doi: 10.1016/j.autrev.2015.11.006. Review.

29. Pisetsky DS. Antinuclear antibodies in healthy people: the tip of autoimmunity's iceberg? Arthritis Res Ther 2011; $13(2): 109$.

30. Fritzler MJ. The antinuclear antibody test: last or lasting gasp? Arthritis Rheum 2011; 63(1): 19-22.

Tables: 0

Figures: 1

References: 30

Received: 08.11.2016

Revised: 17.01.2017

Accepted: 19.01.2017

Address for correspondence:

Barbara Nieradko-Iwanicka, MD, PhD, Assoc. Prof.

Katedra i Zakład Higieny UM

ul. Radziwiłłowska 11

20-080 Lublin

Polska

Tel.: +48 81 448-61-38

E-mail: bnieradkoiwanicka@wp.pl, barbara.nieradko-iwanicka@umlub.pl 